\title{
Nasopharyngeal Colonization and Penicillin Resistance Among Pneumococcal Strains: A Worldwide 2004 Update
}

\author{
Déa M. Cardozo ${ }^{1}$, Cristiana M. C. Nascimento-Carvalho', \\ Fabiane R. Souza ${ }^{2}$ and Nívea M.S. Silva ${ }^{2}$
}

\author{
${ }^{1}$ Department of Pediatrics, Faculty of Medicine, Federal \\ University of Bahia; ${ }^{2}$ Faculty of Biochemistry, Federal University \\ of Bahia; Salvador, BA, Brazil
}

\begin{abstract}
Surveillance of nasopharyngeal pneumococcus has proven to be a valuable tool for the monitoring of antibiotic resistance. We reviewed the latest information on colonization rate and penicillin resistance by making a MEDLINE search, using the terms "nasopharyngeal carriage" and "Streptococcus pneumoniae". Out of 225 articles found, data from 109 recent publications (89\% from 1996-2003) were analyzed. Data were reported from 41 countries of six continents. Individuals under the age of five $(64.3 \%)$ or 10 years $(85.7 \%)$ were enrolled, including children attending day-care centers $(32.1 \%)$ or orphanages $(3.6 \%)$, and healthy individuals $(78.6 \%)$ or sick patients $(43.6 \%)$; biological samples were collected mainly by nasopharyngeal swabs $(89.3 \%)$. The highest colonization rates were reported from Africa (85-87.2\%), where several authors did not find high rates of penicillin resistance. On the other hand, studies conducted in North and Central America reported high-level penicillin resistance at rates of approximately $20-30 \%$. Great variation in the rates of pneumococcal colonization and penicillin resistance were observed within regions or continents. There were also considerable differences in similar populations located in different areas of the same country. Data regarding pneumococcal colonization and penicillin resistance are not available from most countries. We also examined the use of antibiotics to treat pneumococcal infections.
\end{abstract}

Key Words: Streptococcus pneumoniae, nasopharyngeal carriage, penicillin resistance

Penicillin-resistant Streptococcus pneumoniae is an increasing problem worldwide [1]. Nonetheless, surveillance of pneumococcal antibiotic resistance is hampered by the relatively low number of invasive pneumococcal strains that have been isolated [2]. Nasopharyngeal colonization plays an important role in pneumococcal infections [3], and the prevalence of individual clones among isolates from invasive disease has been related to their prevalence in the nasopharynx [4]. In addition, genetic relationships between invasive and nasopharyngeal strains have been found [5].

Pneumococci are part of the normal microbial flora of the nose and pharynx, particularly in young children; they are easily transmitted, usually through droplet secretions, often from an older sibling to a younger sibling and between households within communities [6]. The acquisition of pneumococcus in the nasopharynx occurs early in life, and invasive disease is most likely to occur soon after nasopharyngeal colonization with a newly-acquired strain rather than after long duration of carriage of that strain [7]. Sentinel surveillance of nasopharyngeal pneumococcus has proven to be valuable for the monitoring antibiotic resistance [2] and colonization has been intensively studied in various localities [8].

Received on 2 April 2006; revised 29 June 2006.

Address for correspondence: Dr. Cristiana Nascimento-Carvalho. Rua Prof. Aristides Novis, No. 105 / apto. 1201B, Salvador, Bahia, Brazil, Zip code: 40210-630. Telephone / Fax: 55.71.32357869. Email: nascimentocarvalho@hotmail.com.

The Brazilian Journal of Infectious Diseases 2006;10(4):293-303. (C) 2006 by The Brazilian Journal of Infectious Diseases and Contexto Publishing. All rights reserved.
Our aims in this review were to compile the most recent colonization and penicillin resistance rates reported in each region of the world and to examine the use of antibiotics to treat pneumococcal infection in the face of antibiotic resistance, based on the latest evidence of association of antibiotic use with resistance and of effectiveness of penicillin for the treatment of infection caused by penicillinnonsusceptible pneumococcus (PNSP). PNSP includes strains with resistance to penicillin at an intermediate level (minimal inhibitory concentration [MIC] $0.1 \square 1 \mathrm{Pg} / \mathrm{mL}$ ) as well as strains with high-level resistance to penicillin ( $\mathrm{MIC}>2 \mathrm{Pg} / \mathrm{mL})[2]$.

\section{Search strategy, selection criteria and management of data}

Data for this review were identified by searches of Medline, considering articles published from the 1966 until December 2003. Primary search terms included "nasopharyngeal carriage" and "Streptococcus pneumoniae". All listed articles had their abstracts read, and whenever colonization or penicillin resistance rates were reported, the whole article was read. The data regarding colonization and penicillin resistance were extracted, along with the respective region and time of publication and of performance of the study; the data were analyzed with the statistical software (SPSS version 9.0). The studies conducted within the same region or country were ordered chronologically and the most recent were considered for this analysis. Data regarding the number, age, and the clinical diagnosis of enrolled individuals and biological sample cultures were also collected. Data regarding association of antimicrobial use and pneumococcal resistance to antimicrobials were searched for in the articles selected for 
complete reading, including analysis of the references. Articles reporting results about the effectiveness of penicillin use for the treatment of penicillin-nonsusceptible pneumococcal infections were also searched by using the terms "Streptococcus pneumoniae" and "resistance" and "treatment" in Medline and in the proceedings of international meetings.

\section{Colonization and penicillin resistance studies}

Two-hundred-twenty-five articles were found, out of which $216(96.0 \%)$ were published in English, three (1.3\%) in French, two $(0.9 \%)$ in Italian, and one each $(0.45 \%)$ in Spanish, Japanese, Swedish and Hebrew; the first of those studies was published in 1977. One-hundred-nine articles reported new data about pneumococcal colonization and resistance to penicillin from 41 countries of six continents; $88.9 \%$ of the studies were published as of 1996. The number of studies conducted in each country and the respective frequency were: USA 17 (15.7\%), Israel 12 (11.1\%), France nine (8.3\%), Italy six $(5.5 \%)$, Finland five $(4.6 \%)$, India, Brazil four each $(3 \cdot 7 \%)$, Australia, Greece, Japan, South Africa, Sweden, and Gambia three each (2.8\%), Canada, China, Iceland, Portugal, Taiwan, Turkey, and the United Kingdom two each (1.8\%), and Argentina, Bangladesh, Central Africa Republic, Central and Eastern Europe, Chile, Colombia, Costa Rica, Ecuador, Estonia, Ghana, Indonesia, Malawi, Mexico, Romania, Russia, Switzerland, The Netherlands, Uganda, Vietnam and Zambia, one each $(0.9 \%)$. The most recent data from each region of the world reported in 56 studies are shown in Tables 1-5; 64.3\% and $85.7 \%$ of these studies recruited individuals under the age of five or 10 years and $32.1 \%$ and $3.6 \%$ enrolled children attending day care centers or orphanages, respectively. Biological samples were collected from nasopharyngeal swabs alone $(89.3 \%)$, oropharyngeal swabs alone $(1.8 \%)$ or nasopharyngeal aspirates alone (1.8\%), nasopharyngeal swab or aspirate $(1.8 \%)$, or naso or oral aspirates $(5.3 \%) .42 .8 \%$ of the studies enrolled ill patients including pneumonia $(7 \cdot 3 \%)$, upper respiratory infection $(10.9 \%)$, acute otitis media (3.6\%), and HIV-infected (1.8\%). Healthy individuals were studied in $78.6 \%$ of the investigations. The median interval between collection of samples and publication of results was three years (mean $3+1.2$ years).

Data about pneumococcal carriage and penicillin resistance have not been published for most countries. When data from different countries or regions in the same continent were compared, we found great variation in the pneumococcal carriage and penicillin resistance rates. In South America, the colonization rates varied from $10.0 \%$ (southeast region of Brazil, 2001) [9] to 66.0\% (Quito, Ecuador, 2002) [10] (Table 1); overall penicillin-resistance rates varied from $1.4 \%$ (north region, Brazil, 2001) [9] to 49.0\% (Fortaleza, Brazil, 2002) [11] and highlevel penicillin resistance rates varied from $0 \%$ (Brazil, 2001) $[9,12]$ to $38.8 \%$ (Santa Fé, Argentina, 1997) [13] (Table 1). In
Asia, high-level penicillin resistance was not detected in Blantyre, Malawi, 1997 [17], on Lombok island, Indonesia, 2001 [18] or in Kumamoto, Japan, 2002 [19], whereas it varied from $26 \%$ to $40 \%$ in Hanoi, Vietnan, 2002 [20], Hong Kong, China, 2001 [21] and Taipei, Taiwan, 2003 [22] (Table 2). The highest colonization rates were reported by studies conducted in Africa [23-26], where several authors have not detected high-level penicillin resistance [1, 27-29] (Table 3). In Europe, most of the most-recent studies reported high-level penicillin resistance at a very low frequency, but this was not the case in North and Central America (Tables 4 and 5). The tables summarize the most recent data regarding resistance to penicillin among pneumococcal carriers worldwide. By observing the results from studies in which individuals living in different but nearby cities were assessed, it is also possible to observe differences: in children 0 to 2 years of age, $42 \%$ were colonized with $S$. pneumoniae in Santiago compared to $14 \%$ in Temuco, both of which are Chilean cities [15]; $S$. pneumoniae was isolated from 129 of 187 (69\%) outpatient children in Gaborone and from 53 of 62 (85\%) children in Francistown, two cities in South Africa; this difference was significant $(\mathrm{P}<0.01)$ [30]. Therefore, large differences can exist in similar populations located in different regions of the same country.

The inequality of availability of data regarding pneumococcal colonization and penicillin resistance worldwide is noteworthy (Tables 1-5). In addition to the data presented here, it is well known that resistance to penicillin is very frequent in Spain and Hungary, based on investigations that studied invasive strains [31,32]. Risk factors for pneumococcal colonization have been recognized: these include young age ( $<2$ years old), attending a daycare center, having a lower respiratory tract infection [33,34], as well as risk factors for carriage of $S$. pneumoniae resistant to penicillin: young age $(<2$ years old $)$, and antibiotic use within the previous month [35]. Most (85.7\%) of the studies included in this report enrolled children and several of them were conducted in daycare centers $(32.1 \%)$ or orphanages $(3.6 \%)$.

Almost all children carry $S$. pneumoniae at some time, but only $15 \%$ of all colonized children become infected; this generally occurs within a month of acquisition [7]. For reasons that are not yet completely understood, the balance between the host and these bacteria can be disrupted, resulting in infection [34]. Pneumococcal infection is age-associated, being relatively common in newborns and infants up to two years of age, and much less so in teenaged children and young adults, again increasing in adults older than 65 years [35]. As in most pneumococcal infection cases, antibiotic treatment is initiated empirically, while culture results are pending or even when antibiotic cultures are not feasible; the sentinel surveillance of nasopharyngeal pneumococcus can be used to decide on the first choice of empirical antibiotic treatment for each pneumococcal illness. 


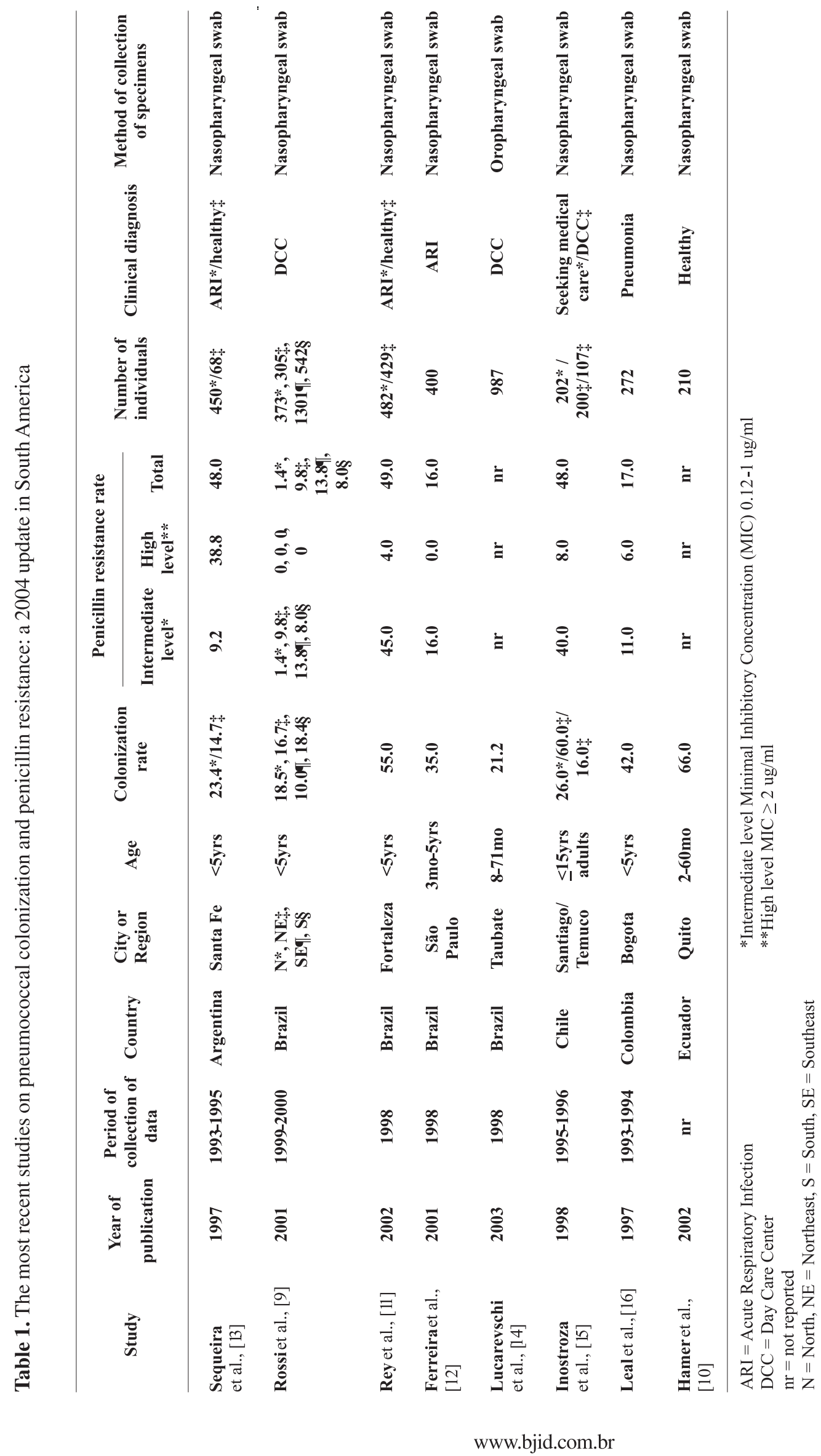




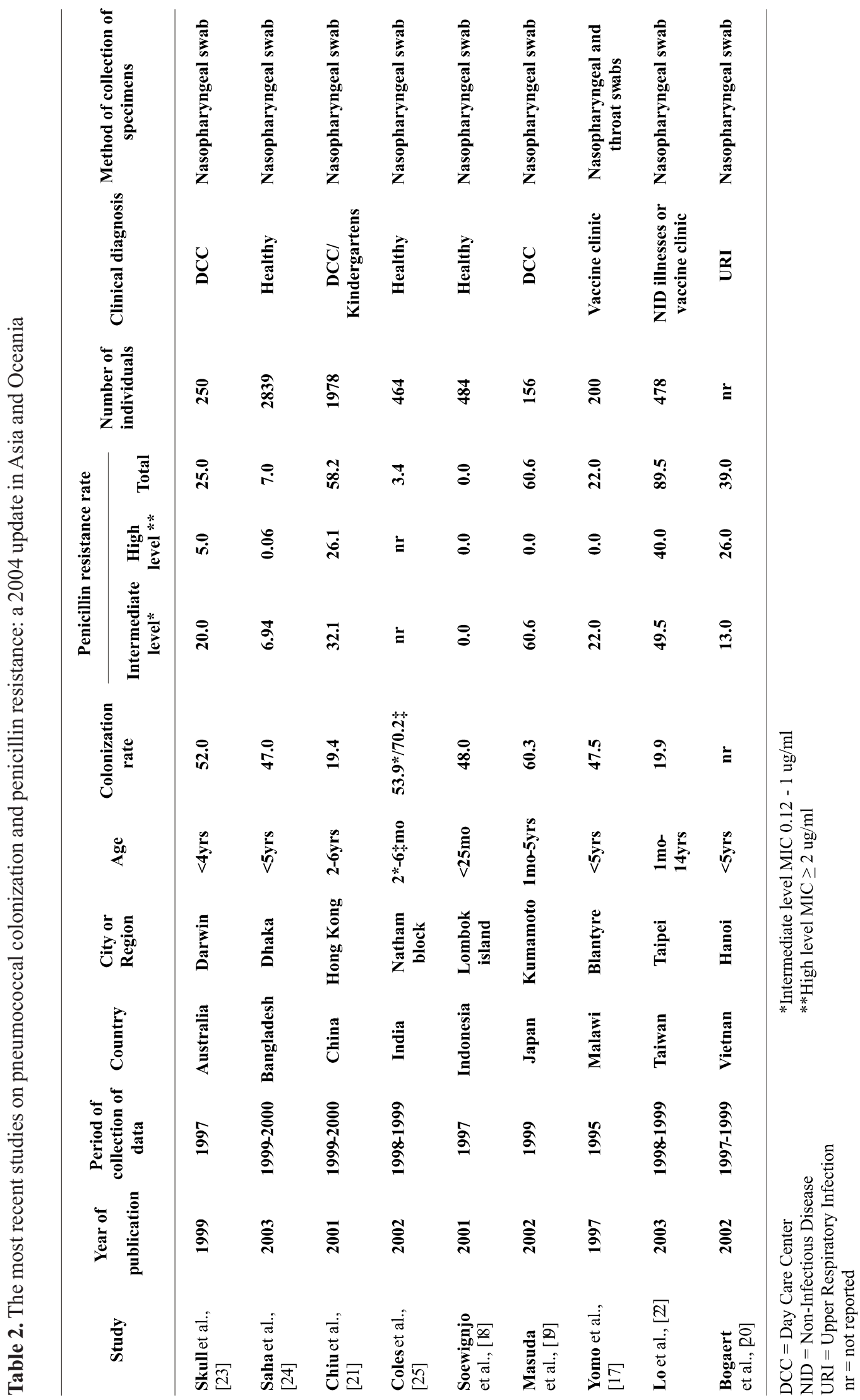




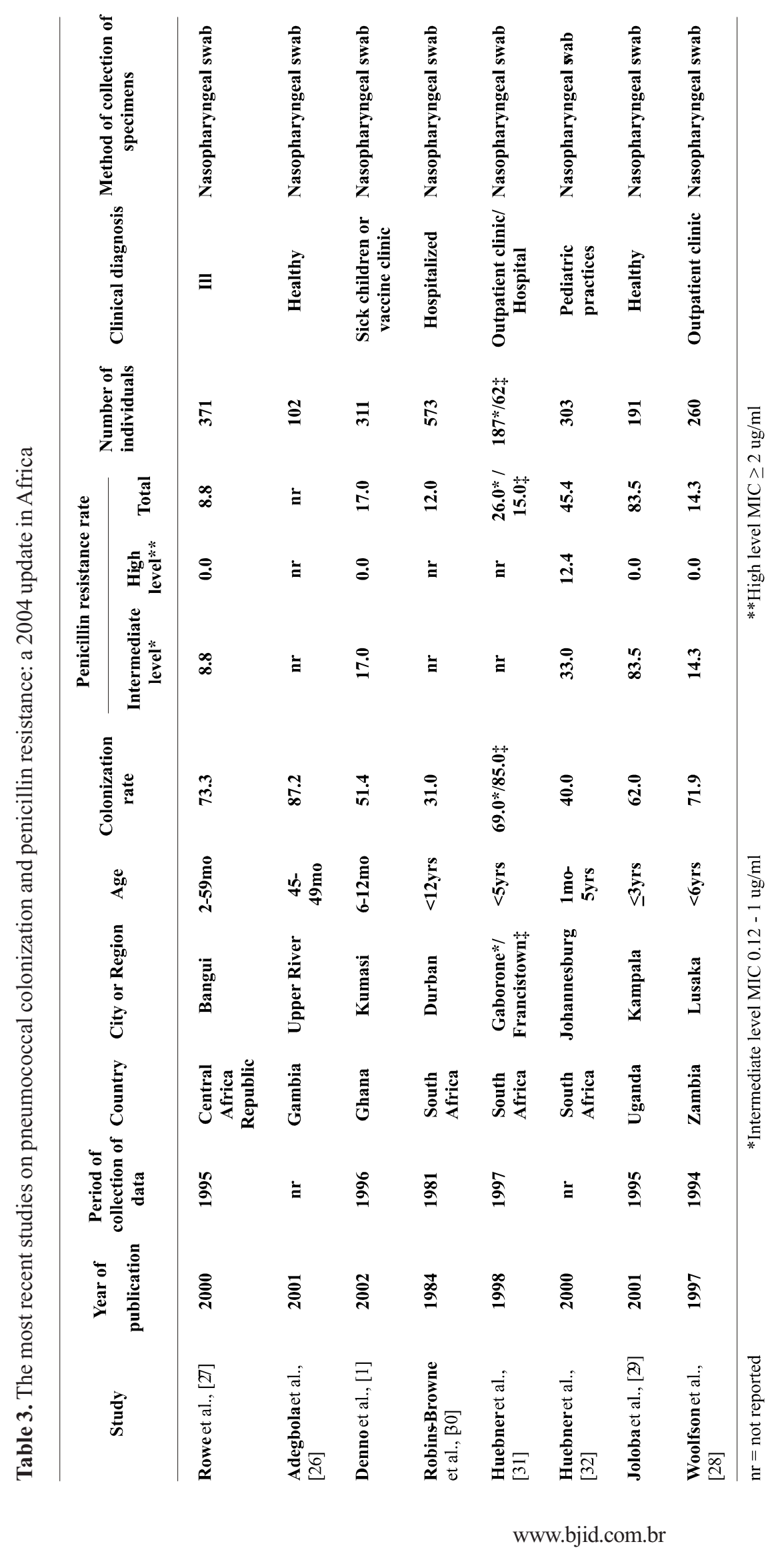




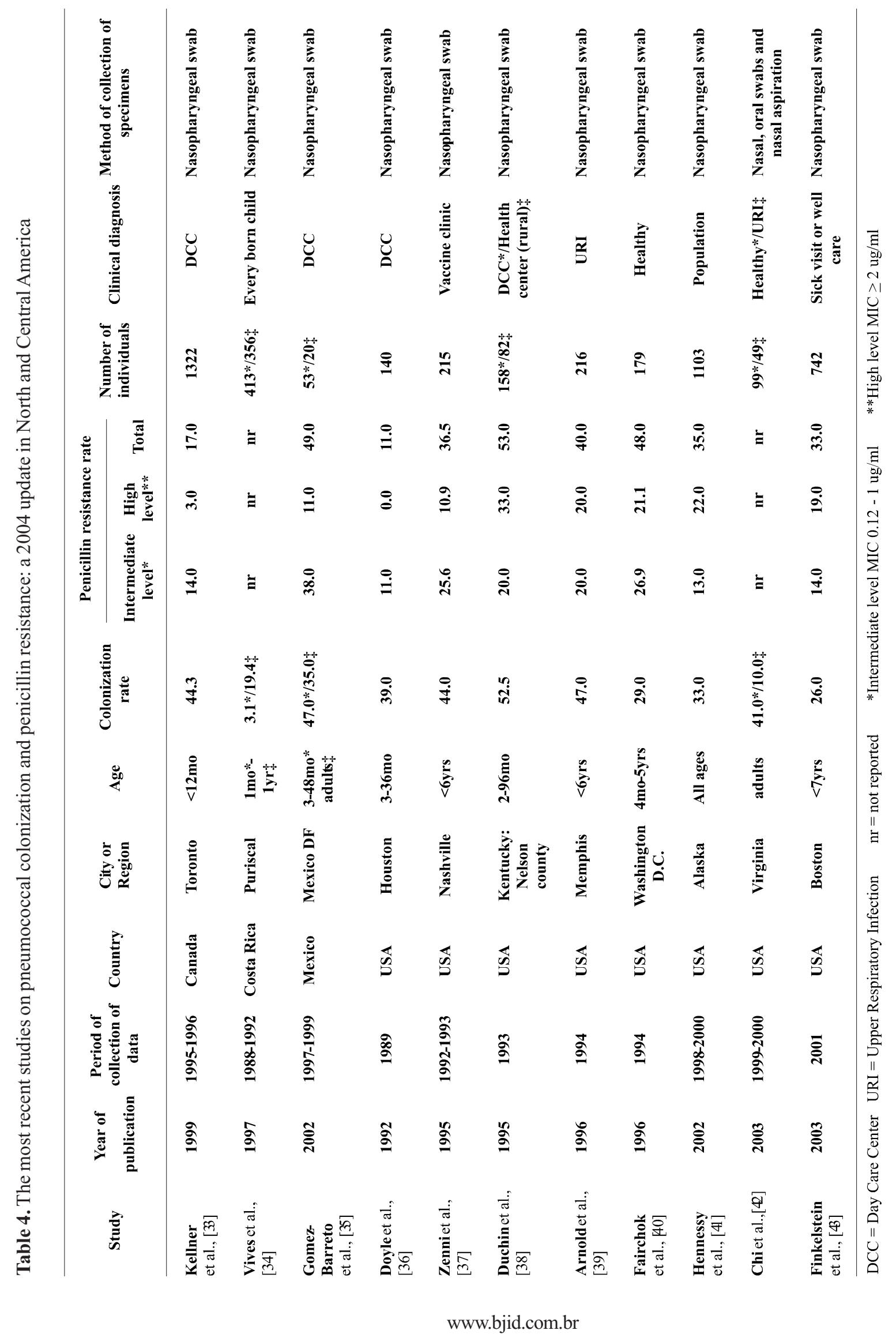




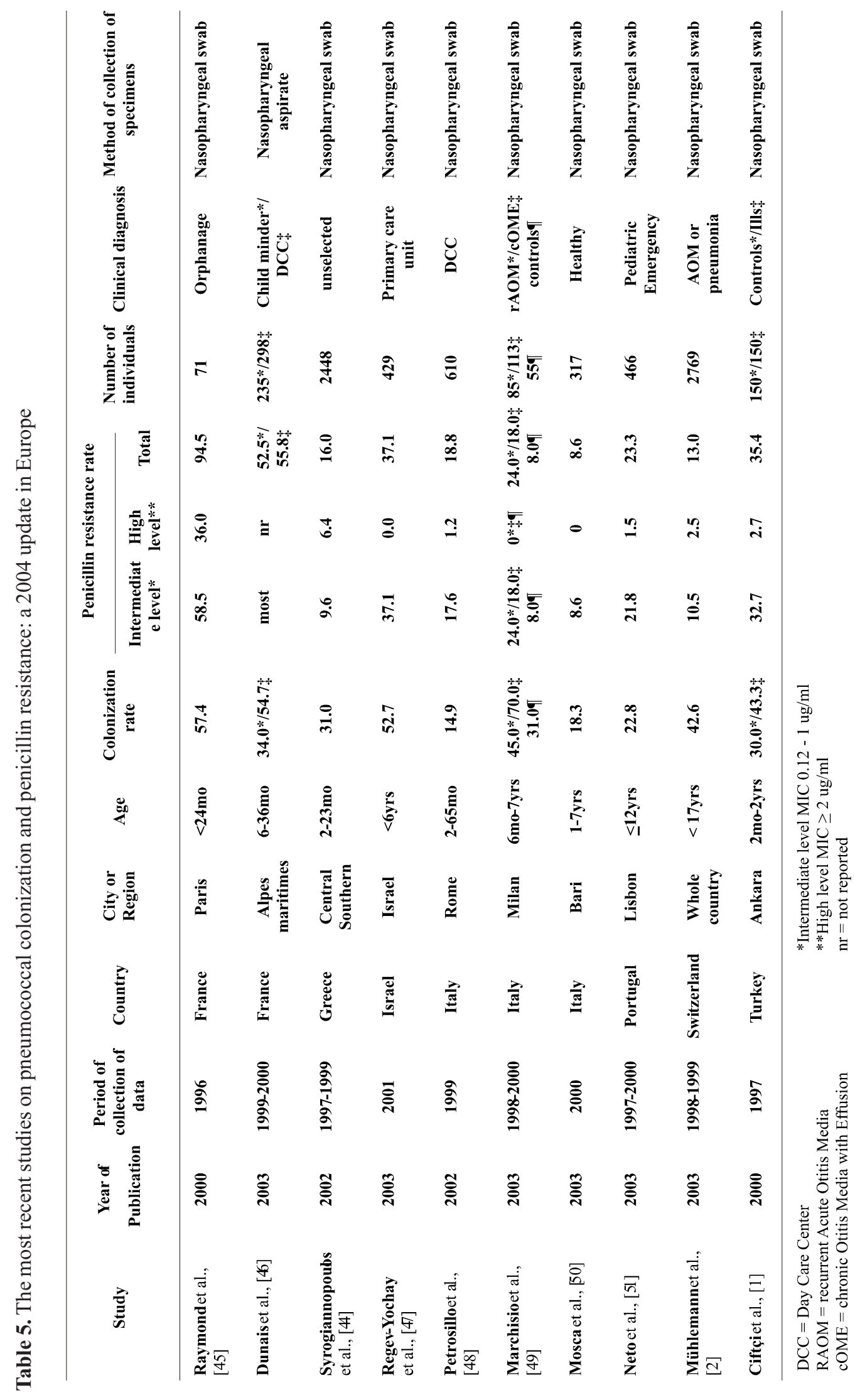




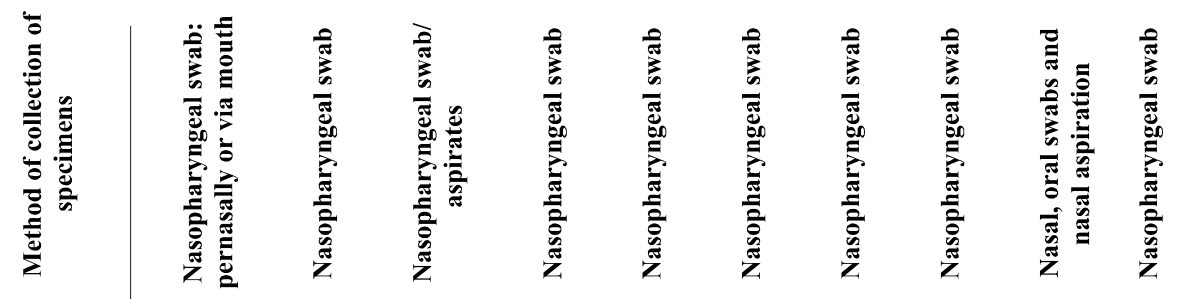

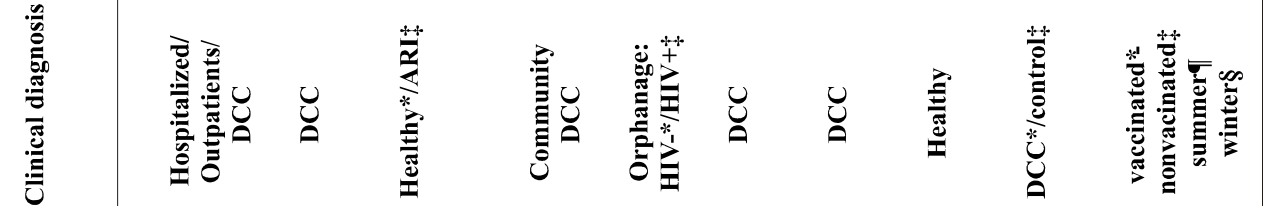

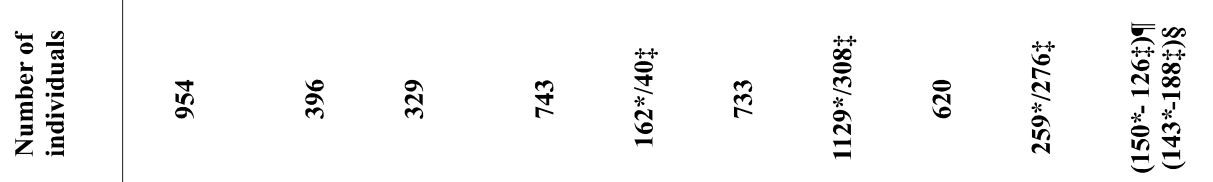

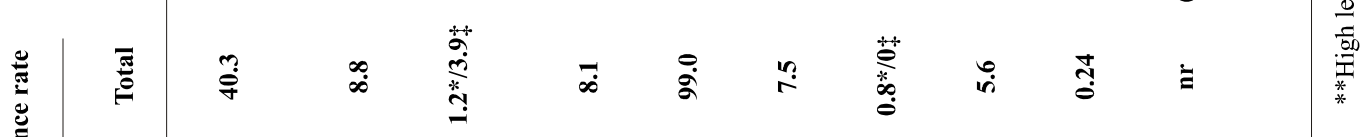

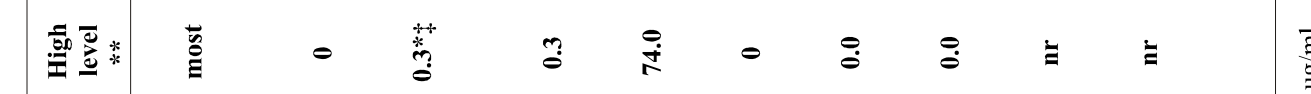

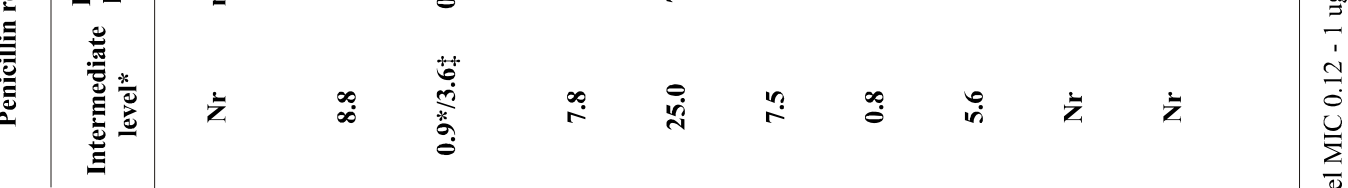

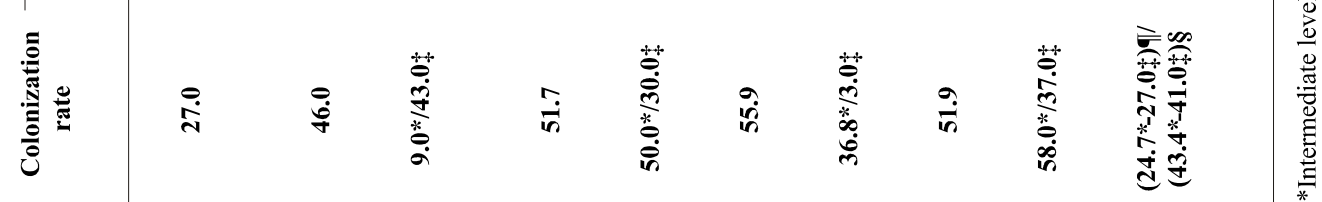

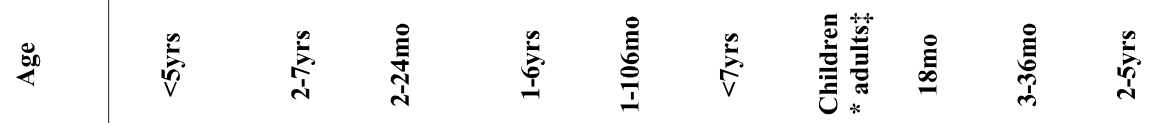

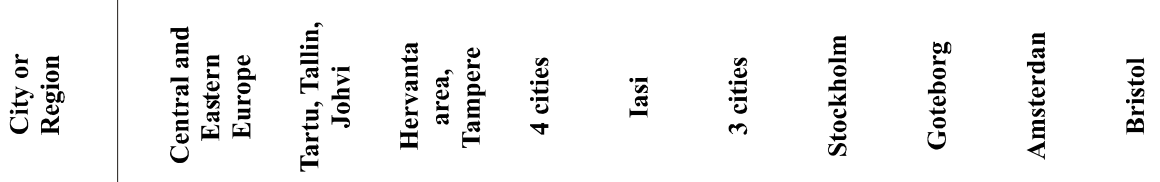

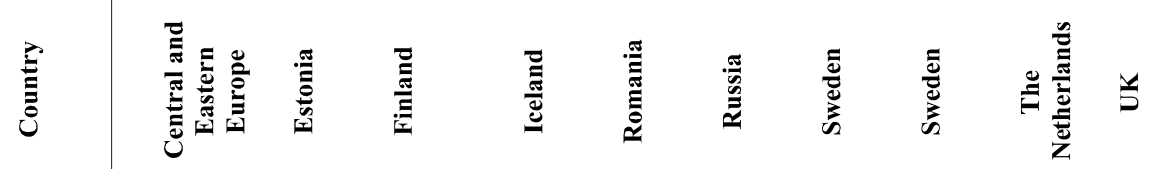

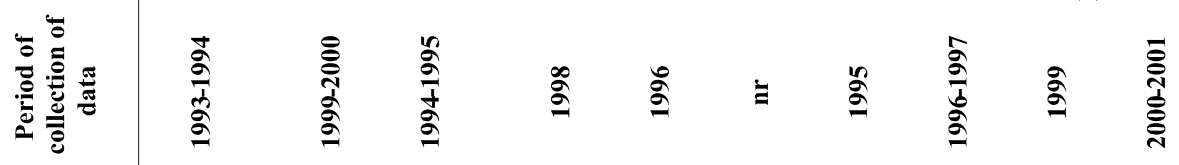

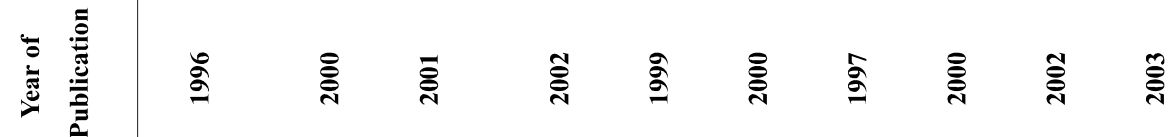

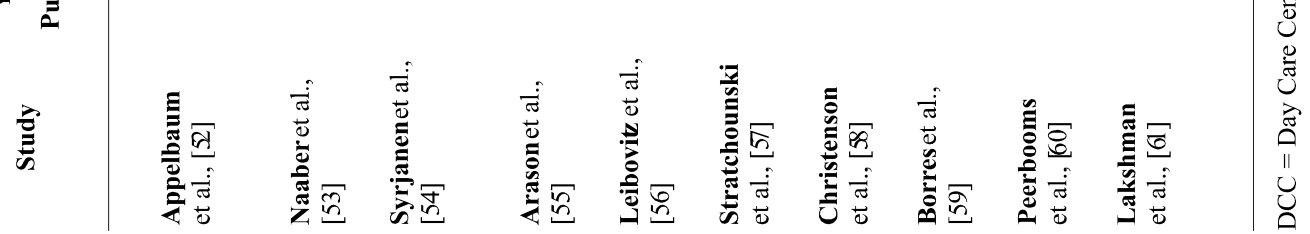


Figure 1. The most recent data regarding frequency of overall and high-level resistance to penicillin* among nasopharyngeal pneumococcal isolates

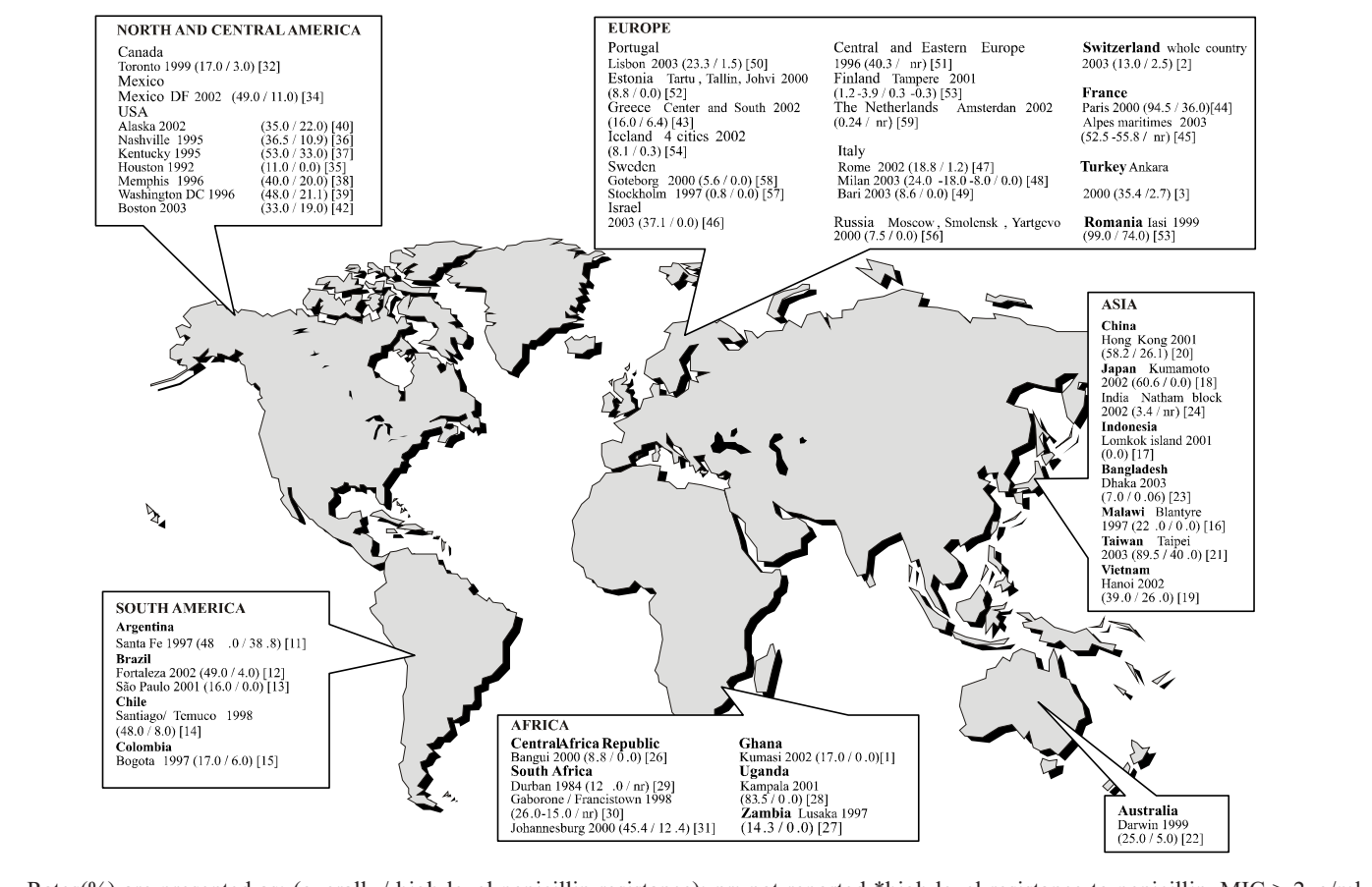

\section{Antimicrobial use and antimicrobial resistance}

Various studies have demonstrated that the frequency of antibiotic use in a community is associated with the frequency of penicillin resistance among pneumococcal strains; the penicillins were the antibiotics that were least associated with this event [36,37-39]. In Iceland, Arason and colleagues [36] studied the prevalence of nasopharyngeal carriage of penicillinresistant pneumococci in children aged under seven years in relation to antimicrobial use (penicillins, cephalosporins, erythromycin and trimethoprim/sulfamethoxazol) in five different communities and found that antimicrobial use, taking into account both individual use and total antimicrobial consumption in the community, was strongly associated with nasopharyngeal carriage of penicillin-resistant pneumococci in children. Hyde and colleagues [37] studied the epidemiology of invasive pneumococcal strains isolated in the USA between 1995 and 1999; they also collected data about the use of macrolides between 1993 and 1999 in the USA; the increase in the rate of resistance to macrolides was correlated with the frequency of macrolide use, those data differed in children aged $<$ versus $>5$ years. Kastner and colleagues [38] studied, once a week, during six weeks, the resistance of pneumococcal nasopharyngeal strains in children before and after receiving different macrolides, in an open, prospective and randomized investigation; they found that in the first week after treatment $90 \%$ of the patients were colonized by resistant strains and the resistance rates returned to baseline numbers by the sixth week for the subgroups that received erythromycin, clarithromycin, roxithromycin and josamycin, but this was not the case for the group that received azythromycin $(\mathrm{P}<0.005)$. Garcia-Rey and colleagues [39] studied 1,684 pneumococcal strains isolated from patients with community-acquired respiratory infection; they collected data about the frequency of use of different antimicrobials from distinct communities in Spain and found a correlation between antimicrobial use and pneumococcal resistance. When different antimicrobials were examined separately by multivariate analysis, aminopenicillins, cephalosporins and macrolides were found to be correlated with resistance at increasing rates, respectively.

\section{Antimicrobial resistance and treatment with antimicrobials}

Several investigators have given evidence that high doses of amoxicillin [40] or penicillin G [41] are effective for the treatment of respiratory infections caused by high-level penicillin resistant pneumococcal strains [40,41]. Piglansky and colleagues [40] evaluated by tympanocentesis, at enrollment and on days four to six of therapy, 50 culturepositive patients aged 3-22 months with acute otitis media (AOM); the patients received amoxicillin $(80 \mathrm{mg} / \mathrm{kg} / \mathrm{day})$ and susceptibility to penicillin was assessed by the E-test. Twentyfour isolates of $S$ pneumoniae were recovered, out of which 18 were nonsusceptible to penicillin (two of 18 were highly resistant to penicillin). Eradication was achieved in $92 \%$ of the patients with pneumococcal AOM. The authors concluded 
that the overall clinical efficacy of amoxicillin was good. Moreover, the predominant pathogens isolated from children with AOM failing high dose amoxicillin therapy were betalactamase-producing organisms; specifically Haemophilus influenzae. In a study conducted by Agosti and colleagues [71], 269 children aged 3-59 months were hospitalized due to severe pneumonia caused by $S$. pneumoniae recovered from blood or pleural fluid; each of those patients received intravenous penicillin $(200,000 \mathrm{IU} / \mathrm{kg} /$ day $)$. The pneumococcal isolates were classified as susceptible (131), intermediate resistant (66), highly resistant (53) or unknown (19) to penicillin; there was no association between pneumonia caused by nonsusceptible pneumococcus and failure of penicillin treatment. Various experts have recommended the use of penicillin to empirically-treat pneumococcal infections that do not compromise the central nervous system [42-45]. In the case of pneumococcal meningitis, it is clear that penicillin treatment is contraindicated for isolates with intermediate or high-level resistance [45]. A number of clinical studies of pneumococcal pneumonia have assessed the treatment outcomes following infection by drug resistant pneumococcus, compared with the course of infection by drug-susceptible pneumococcus isolates in the same population [45]. Echoing the results of previous studies, older age and underlying disease, but not drug resistance in the isolate, were found to be the most important predictors of mortality from pneumococcal pneumonia [45].

In a recent cross-sectional study conducted in St. Louis, USA, S. pneumoniae was isolated from the nasopharynx of $85(40 \%)$ of 212 patients younger than seven years who had AOM, nonspecific upper respiratory infection, cough, acute sinusitis, or pharyngitis; 41 (48\%) of 85 isolates were PNSP and $6(7 \%)$ were nonsusceptible to amoxicillin (NSSP-A), and among the 212 study patients the prevalence of PNSP was $19 \%$ and of NSSP-A was 3\%. Carriage of NSSP was increased in child-care attendees compared with nonattendees; based on these data the authors recommended to treat most children who have uncomplicated AOM with standard-dose amoxicillin, while children who attend child care centers or who have recently been treated with antibiotics may require treatment with high-doses of amoxicillin [46].

Global containment of antimicrobial resistance is a matter of concern for authorities, as spread of resistance may be influenced by interpersonal contact promoted by travelers crossing national boundaries [47]. Based on the foregoing evidence, we propose that pneumococcal infection that does not compromise the central nervous system be treated empirically by using penicillin, worldwide $[42,46,48]$. This routine may reduce the frequency of pneumococcal penicillin resistance and therefore minimize the problem of treating severe pneumococcal infection caused by resistant strains, especially when the central nervous system is compromised.

\section{Conclusion}

Pneumococcal penicillin resistance varies considerably from one region to another around the world. The penicillins have been the antibiotics least associated with pneumococcal antibiotic resistance [36,37-39]; there is no evidence of failure of penicillin treatment at high doses in resistant pneumococcal infections that do not compromise the central nervous system $[41,46,48]$. Therefore, it is possible that the routine use of penicillin to treat pneumococcal infections that does not compromise the central nervous system will diminish the frequency of pneumococcal penicillin resistance.

\section{References}

1. Denno D.M., Frimpong E., Gregory M., Steele R.W. Nasopharyngeal carriage and susceptibility patterns of Streptococcus pneumoniae in Kumasi, Ghana. West Afr J Med 2002;21:233-6.

2. Muhlerman K., Matter H.C., Tauber M.G., Bodmer T. Nationwide surveillance of nasopharyngeal Streptococcus pneumoniae isolates from children with respiratory infection, Switzerland, 1998-1999. J Infect Dis 2003;187:589-96.

3. Ciftçi E., Dogru U., Aysev D., et al. Nasopharyngeal colonization with penicillin-resistant Streptococcus pneumoniae in Turkish children. Pediatr Int 2000;42:552-6.

4. Brueggemann A.B., Griffths D.T., Meats E., et al. Clonal relationship between invasive and carriage Streptococcus pneumoniae and serotype- and clone-specific differences in invasive disease potential. J Infect Dis 2003; 187:1424-32.

5. de Andrade A.L., Pimenta F.C., Brandileone M.C., et al. Genetic relationship between Streptococcus pneumoniae isolates from nasopharyngeal and cerebrospinal fluid of two infants with pneumococcal meningitis. J Clin Microbiol 2003;41:3970-2.

6. O'Brien K.L., Nohynek H., and the WHO Pneumococcal Vaccine Trials Carriage Working Group. Report from a WHO Working Group: standard method for detecting upper respiratory carriage of Streptococcus pneumoniae. Pediatr Infect Dis J 2003;22:133-40.

7. Gray B.M., Converse G.M., Dillon H.C. Epidemiologic studies of Streptococcus pneumoniae in infants: acquisition, carriage and infection during the first 24 months of life. J Infect Dis 1980; $142: 923-33$.

8. Garcia-Rodriguez J.A., Fresnadillo Martínez M.J. Dynamics of nasopharyngeal colonization by potential respiratory pathogens. J Antimicrob Chemother 2002;50 Suppl S2:59-73.

9. Rossi F., Andreazzi D., Maffucci M., Pereira AA. Susceptibility of $S$. pneumoniae to various antibiotics among strains isolated from patients and healthy carriers in different Regions of Brazil (1999-2000). Braz J Infect Dis 2001;5:305-12.

10. Hamer D.H., Egas J., Estrella B., et al. Assessment of the Binas NOW Streptococcus pneumoniae urinary antigen test in children with nasopharyngeal pneumococcal carriage. Clin Infect Dis 2002;34:1025-8.

11. Rey L.C., Wolf B., Moreira L.B., et al. Antimicrobial susceptibility and serotypes of nasopharyngeal Streptococcus pneumoniae in children with pneumonia in children attending day-care centers in Frotaleza, Brazil. Int J Antimicrob Agents 2002;20:86-92.

12. Ferreira L.L.M., Carvalho E.S., Berezin E.N., Brandileone M.C. Nasopharyngeal colonization and antimicrobial resistance of Streptococcus pneumoniae isolated from children with acute rhinopharyngitis. J Pediatr (Rio J.) 2001;77:227-34.

13. Sequeira M.D., Zerbini E., Imaz M.S., et al. Etiology of acute lower respiratory infections among children younger than 5 years old in Santa Fe. Medicina (B Aires) 1997;57:191-9. 
14. Lucarevschi B.R., Baldacci E.R., Bricks L.F., et al. Oropharyngeal carriage of Streptococcus pneumoniae by children attending day care centers in Taubaté, SP: correlation between serotypes and conjugated heptavalent pneumococcal vaccine. J Pediatr (Rio J.) 2003;79:215-20.

15. Inostroza J., Trucco O., Prado V., et al. Capsular serotype and antibiotic resistance of Streptococcus pneumoniae isolates in two Chilean cities. Clin Diagn Lab Immunol 1998;5:176-80.

16. Leal A.L., Castañeda E. Susceptibilidad antimicrobiana de Streptococcus pneumoniae colonizante de nasofaringe en niños colombianos com neumonía. Rev Panam Salud Publica 1997;1: 266-72.

17. Yomo A., Subramanyam V.R., Fudzulani R., et al. Carriage of penicillin-resistant pneumococci in Malawian children. Ann Trop Pediatr 1997; 7:239-43.

18. Soewignjo S., Gessner B.D., Sutanto A., et al. Streptococcus pneumoniae nasopharyngeal carriage prevalence, serotype distribution, and resistance patterns among children on Lombok island, Indonesia. Clin Infect Dis 2001;32:1039-43.

19. Masuda K., Masuda R., Nnishi J., et al. Incidences of nasopharyngeal colonization of respiratory bacterial pathogens in Japanese children attending day-care centers. Pediatr Int 2002;44:376-80.

20. Bogaert D., Ha N.T., Sluijter M., et al. Molecular epidemiology of pneumococcal carriage among children with upper respiratory tract infections in Hanoi, Vietnam. J Clin Microbiol 2002;40:3903-8.

21. Chiu S.S., Ho P.L., Chow F.K.H., et al. Nasopharyngeal carriage of antimicrobial-resistant Streptococcus pneumoniae among young children attending 79 kindergartens and day care centers in Hong Kong. Antimicrob Agents Chemother 2001;45:276570.

22. Lo W.T., Wang C.C., Yu C.M., Chu M.L. Rate of nasopharyngeal carriage, antimicrobial resistance and serotype of Streptococcus pneumoniae among children in northern Taiwan. J Microbiol Immunol Infect 2003;36:175-81.

23. Adegbola R.A., Obaro S.K., Biney E., Greenwood B.M. Evaluation of Binax now Streptococcus pneumoniae urinary antigen test in children in a community with a high carriage rate of pneumococcus. Pediatr Infect Dis J 2001;20:718-9.

24. Rowe A.K., Deming M.S., Schwartz B., et al. Antimicrobial resistance of nasopharyngeal isolates of Streptococcus pneumoniae and Haemophilus influenzae from children in the Central African Republic. Pediatr Infect Dis J 2000;19:43844.

25. Woolfson A., Huebner R., Wasas A., et al. Nasopharyngeal carriage of community-acquired, antibiotic-resistant Streptococcus pneumoniae in a Zambian paediatric population. Bull World Health Organ 1997;75:453-62.

26. Joloba M.L., Bajaksouzian S., Palavecino E., et al. High prevalence of carriage of antibiotic-resistant Streptococcus pneumoniae in children in Kampala Uganda. Int J Antimicrob Agents 2001; $17: 395-400$

30. Huebner R.E., Wasas A., Mushi A., et al. Nasopharyngeal carriage and antimicrobial resistance in isolates of Streptococcus pneumoniae and Haemophilus influenzae type $\mathrm{b}$ in children under 5 years of age in Botswana. Int J Infect Dis 1998; 3 : $18-25$.

31. Fenoll A., Bourgon C., Munoz R., et al. Serotype distribution and antimicrobial resistance of Streptococcus pneumoniae isolates causing systemic infections in Spain, 1979-1989. Rev Infect Dis 1991; 13: 56-60.
32. Marton A., Gulayas M., Munoz R., Tomasz A. Extremely high incidence of antibiotic resistance in clinical isolates of Streptococcus pneumoniae in Hungary. J Infect Dis 1991; 163: $542-8$.

33. Neto A.S., Lavado P., Flores P., et al. Risk factors for the nasopharyngeal carriage of respiratory pathogens by portuguese children: phenotype and antimicrobial susceptibility of Haemophilus influenzae and Streptococcus pneumoniae. Microb Drug Resist 2003; 9: 99-108.

34. Ghaffar F., Friedland I.R., McCracken G.H. Dynamics of nasopharyngeal colonization by Streptococcus pneumoniae. Pediatr Infect Dis J 1999; 18: 638-46.

35. Arason V.A., Kristinsson K.G., Sigurdson J.A., et al. Do antimicrobials increase the carriage of penicillin resistant pneumococci in children? Cross sectional prevalence study. BMJ 1996; 313: 387-91.

36. Burman L.A., Norrby R., Trollfors B. Invasive pneumococcal infections: incidence, predisposing factors, and prognosis. Rev Infect Dis 1985; 7: 133-42.

37. Hyde T.B., Gay K., Stephens D.S. Macrolide resistance among invasive Streptococcus pneumoniae isolates. JAMA 2001; 286:1857-62.

38. Kastner U., Guggeenbichler J.P. Influence of macrolide antibiotics on promotion of resistance in the oral flora of children. Infection 2001; 29: 251-6.

39. Garcia-Rey C., Aguilar L., Baquero F., et al. Importance of local variations in antibiotic consumption and geographical differences of erythromycin and penicillin resistance in Streptococcus pneumoniae. J Clin Microbiol 2002; 40: 159-64.

40. Piglansky L., Leibovitz E., Raiz S., et al. Bacteriologic and clinical efficacy of high dose amoxicillin for therapy of acute otitis media in children. Pediatr Infect Dis J 2003; 22: 405-13.

41. Agosti M.R., Benguigui Y., Berezin E.W., et al. Penicillin is effective for treatment of pneumonia due to penicillin nonsusceptible Streptococcus pneumoniae. [Abstract AM040] In: Abstracts of the $2^{\text {nd }}$ International Conference on Improving Use of Medicines (Chiang Mai) Chiang Mai: Thailand, March 30 to April 2, 2004.

42. Friedland I.R., McCracken G.H., Jr. Management of infections caused by antibiotic-resistant Streptococcus pneumoniae. N Engl J Med 1994; 331: 377-82.

43. Klugman K.P., Friedland I.R. Antibiotic-resistant pneumococci in pediatric disease. Microb Drug Resist 1995; 1: 5-8

44. Klugman K.P. Bacteriological evidence of antibiotic failure in pneumococcal lower respiratory tract infections. Eur Respir J Suppl 2002; 36: 3S-6S.

45. Bishai $\mathrm{W}$. The in vivo-in vitro paradox in pneumococcal respiratory tract infections. J Antimicrob Chemother 2002; 49: 433-6.

46. Garbutt J, St Geme JW $3^{\text {rd }}$, May A, Storch GA, Shackelford PG. Developing community-specific recommendations for first-line treatment of acute otitis media: is high-dose amoxicillin necessary? Pediatrics 2004; 114: 342-7.

47. WHO. WHO Global Strategy for Containment of Antimicrobial Resistance. WHO/CDS/CSR/DRS/2001.2. Geneva: World Health Organization, 2001.

48. Heffelfinger JD, Dowell SF, Jorgensen JH et al. Management of community-acquired pneumonia in the era of pneumococcal resistance. Arch Intern Med 2000; 160: 1399-408. 